Harin Sellahewa and Sabah A. Jassim, "Image quality-based adaptive illumination normalisation for face recognition", Proc. SPIE 7306, Optics and Photonics in Global Homeland Security V and Biometric Technology for Human Identification VI, 73061V (May 05, 2009); doi:10.1117/12.819087; http://dx.doi.org/10.1117/12.819087

Copyright 2009 Society of Photo Optical Instrumentation Engineers. One print or electronic copy may be made for personal use only. Systematic reproduction and distribution, duplication of any material in this paper for a fee or for commercial purposes, or modification of the content of the paper are prohibited." (http://spie.org/x1125.xml) 


\title{
Image Quality-based Adaptive Illumination Normalisation for Face Recognition
}

\author{
Harin Sellahewa and Sabah A. Jassim \\ Department of Applied Computing, \\ The University of Buckingham, Buckingham, MK18 1EG, UK
}

\begin{abstract}
Automatic face recognition is a challenging task due to intra-class variations. Changes in lighting conditions during enrolment and identification stages contribute significantly to these intra-class variations. A common approach to address the effects such of varying conditions is to pre-process the biometric samples in order normalise intra-class variations. Histogram equalisation is a widely used illumination normalisation technique in face recognition. However, a recent study has shown that applying histogram equalisation on well-lit face images could lead to a decrease in recognition accuracy. This paper presents a dynamic approach to illumination normalisation, based on face image quality. The quality of a given face image is measured in terms of its luminance distortion by comparing this image against a known reference face image. Histogram equalisation is applied to a probe image if its luminance distortion is higher than a predefined threshold. We tested the proposed adaptive illumination normalisation method on the widely used Extended Yale Face Database B. Identification results demonstrate that our adaptive normalisation produces better identification accuracy compared to the conventional approach where every image is normalised, irrespective of the lighting condition they were acquired. Keywords: Biometrics, Quality Measures, Face Recognition, Illumination, Wavelet Transforms
\end{abstract}

\section{INTRODUCTION}

Automatic face recognition is one of the most desired forms of person identification. Continued research in this area is motivated by the need for convenient, reliable, pervasive and universal person identification methods to grant access to information and services and as a counter measure to identity theft. Robust face recognition algorithms, complemented by the availability of high quality sensors at a low-cost, have made it possible to transfer face biometrics from theory to successful, automated person identification systems for various applications. Most common approaches to face recognition are appearance based, holistic methods such as eigenfaces, ${ }^{1}$ Fisher's Linear Discriminant (FLD) ${ }^{2,3}$ and Independent Component Analysis (ICA), ${ }^{4}$ which treat face images as a point in a highdimensional image space and geometrical feature points based approaches. ${ }^{5,6}$ However, automatic face recognition remains a challenging task when presented with uncooperative users as well as in uncontrolled environments. Intra-class variations due to occlusion, facial expressions, changes in head pose, orientation and lighting conditions are some of the difficulties an automated face recognition system is faced with, resulting in increased identification errors. These variations can be thought of as distortions from "standard" reference images, giving rise to measures of face image quality. ${ }^{7-9}$ This paper is concerned with face recognition under varying illumination conditions.

Changes in lighting conditions during enrolment and identification stages contribute significantly to intraclass variations. Typical methods used to address varying illumination conditions could be categorised as: feature-based methods, generative methods and holistic methods. In feature-based methods, faces are represented by illumination invariant features. These are geometrical measurements and relationships between local facial features such as the eyes, mouth, nose and chin.5,6 Feature-based methods are known to be robust against varying illumination conditions. However, they rely on accurate automated face and facial feature detection, which are challenging tasks on their own, especially under extreme lighting conditions. 
Send correspondence to Harin Sellahewa

Harin Sellahewa: E-mail: harin.sellahewa@buckingham.ac.uk Sabah A.

Jassim: E-mail: sabah.jassim@buckingham.ac.uk

Generative methods ${ }^{10-13}$ have been proposed to address the problem of varying illumination conditions based on the assumption of the Lambertian model. Previous work has demonstrated that the variability of images under a fixed pose, consisting of only diffuse reflection components and varying illumination conditions can be represented by a linear combination of three basis images. ${ }^{14,15} \operatorname{In}^{16}$ Belhumeur and Kriegman demonstrated that a set of images of an object under fixed posed, consisting of diffuse reflection components and shadows under arbitrary lighting conditions forms a convex cone (termed the illumination cone) in the image space and that this illumination cone can be approximated by a low-dimensional subspace. Experimental results have shown that these generative methods perform well under varying illumination conditions. However, generative approaches require a number of training samples which represent extreme illumination conditions to model the illumination cone for a given face. It may be possible to acquire sufficient number of training images for certain applications such as ID cards and physical access control systems, where the person under consideration is cooperative, but it is not so for surveillance and counter terrorism related applications.

In holistic methods, the entire face image is considered for face representation without taking into account any specific geometrical features. A face image is considered as a point in a high-dimensional image space. To avoid computational complexities and to reduce redundant data, a face image is first linearly transformed into a lowdimensional subspace, prior to extracting a feature vector that would be used later for classification. The most commonly used dimension reduction technique in face recognition is the Principal Component Analysis (PCA). ${ }^{1}$ PCA is known to retain intra-class variations due to changes in illumination and pose. It has been demonstrated that leaving out the first 3 eigenfaces that corresponds to the 3 most significant eigenvalues could reduce the effect of variations in illumination. ${ }^{2}$ However, this may also lead to the loss of discriminant information that is useful for accurate identification. An alternative approach to PCA based linear projection is Fisher's Linear Discriminant (FLD), or the Linear Discriminant Analysis (LDA) which is used to maximize the ratio of the determinant of the interclass scatter to that of intra-class scatter. ${ }^{2,3}$ Like generative methods, the downside of these approaches is that a number of samples, of each person, from different conditions are required to train the subspace in order to identify faces in uncontrolled environments. In addition, adding new identities to the recognition system is cumbersome as it requires to retrain the face subspace. In recent years, wavelet transforms (WT) have been successfully used in a variety of face recognition schemes as a dimension reduction technique ${ }^{17,18}$ or as a tool to extract a multi-resolution feature representation of a given face image. ${ }^{19,20}$ In most instances, only the approximation subband of the WT are used to represent face images as these give the best overall recognition accuracy. The detail subbands (i.e. horizontal, vertical and diagonal features) of the transformed face images are generally ignored.

A common approach to address the effects of varying lighting conditions is to pre-process face images in order to normalise such variations. Histogram equalisation (HE) is a commonly used technique in holistic methods to normalise the illumination. In automated face recognition systems, such normalisation techniques are applied on all face image samples during enrolment and testing stages. However, previously in, ${ }^{21}$ we have shown that normalisation by HE could lead to a decrease in face recognition accuracy when images are captured under reasonably controlled lighting conditions. In this paper, an adaptive approach to illumination normalisation, based on face image quality, is presented.

The contribution in this paper is two fold. First, the illumination quality of a given probe face image is measured in terms of its luminance distortion compared to a known reference image. This quality measure is used as the base for adapting the application of illumination normalisation procedures. Secondly, the global quality measure is extended to a region-based quality measure which is then used for a region quality-based approach to adaptive illumination normalisation. The work presented in this paper is an initial step of a wider study on sample quality based adaptive approaches for face recognition, which aims to address the challenges posed by varying conditions in real-life face recognition systems.

The rest of the paper is organised as follows. Section. 2 presents a review of recent work in ${ }^{21}$ and highlight the need for an adaptive approach to illumination normalisation. We discuss the use of biometric sample quality 
measures and introduce the illumination quality measure used in this study in Sec. 3. Section 4 is devoted to the description of the proposed adaptive approach to illumination normalisation and the experiments conducted to compare the identification performance of the proposed adaptive method with the conventional approach to illumination normalisation. A region quality-based adaptive approach to illumination normalisation is presented in Sec. 5. Finally, conclusions and future work are presented in Sec. 6.

\section{BACKGROUND}

One of the main influencing factors on the accuracy of automatic face recognition systems is the intra-class variations due to changes in lighting conditions between the enrolled (gallery) and test (probe) images. These changes can be attributed to the differences in light source, direction and intensity of light. Common approaches to illumination normalisation methods include HE, histogram matching (HM) and gamma intensity correction (GIC) and Self Quotient Image (SQI). ${ }^{22}$ It has been shown that these normalisation techniques help to improve recognition accuracy. ${ }^{23,24}$ The affect of varying lighting conditions on different wavelet-based face representations was analysed in $^{21}$ using the popular Yale Face Database B ${ }^{11}$ (YaleB) and Extended Yale Face Database B ${ }^{13}$ (Extended YaleB). Contrary to expectation, the results showed that the use of HE on images with small variations in illumination conditions lead to a decrease in recognition accuracy. Here we will discuss relevant work in, ${ }^{21}$ beginning with a brief introduction to wavelet-based face recognition.

\subsection{Wavelet Transforms in Face Recognition}

A wavelet transform (WT) is a multiresolution signal analysis tool that hierarchically decomposes a signal into its low- and high-frequency components allowing one to view the signal's regular patterns as well as its anomalies. The discrete wavelet transform (DWT) is a special case of the WT that provides a compact representation of a signal in time and frequency that can be computed efficiently. ${ }^{25,26}$

There are a number of different ways of applying a 2D-wavelet transform. The most commonly used wavelet decomposition of an image, adopted in this paper, is the pyramid scheme (also referred to as the non-standard decomposition). At a resolution level of $k$, the pyramid scheme decomposes an image $I$ into $3 k+1$ subbands ( $L L_{k}, H L_{k}, L H_{k}, H H_{k}, . ., H L_{1}, L H_{1}, H H_{1}$ ), with $L L_{k}$, being the lowest-pass subband. The subbands $L H_{1}$ and $H L_{1}$, contain finest scale wavelet coefficients that get coarser with $L L_{k}$ being the coarsest. The $L L_{k}$ subband is considered as the $k$-level approximation of $I$, while $H L_{k}, L H_{k}$, and $H H_{k}$ captures vertical, horizontal and diagonal features of the image $I$.

A subband of a wavelet transformed face image can be used as face feature descriptor. 18,19,27 Therefore, different decomposition levels and/or wavelet filters yield different face feature vectors. Two such feature vectors are typically compared by calculating a distance score (e.g. using CityBlock distance) and the probe's identity is classified according to the nearest-neighbour. Throughout this paper, the Haar wavelet filter is used for the DWT and the subband coefficients are normalised by z-score (ZN) normalisation. The CityBlock distance is used to calculate a distance score between a probe and a gallery image.

\subsection{Experiment Data: Extended Yale Face Database B}

Experiments in ${ }^{21}$ used the cropped face images of the Extended YaleB. ${ }^{13}$ The Extended YaleB consists of 38 subjects. Each subject was imaged under 64 illumination conditions, all in frontal pose, capturing a total of 2414 images. In addition to these images, an image was captured under ambient illumination for each one of the subjects. This image was not used for the experiments.

The images in the Extended YaleB are divided into 5 illumination subsets according to the angle $\theta$ of the lightsource with respect to the optical axis of the camera. The subsets and the number of images used in each subset are shown in Tab. 1. All 168×192 cropped images were resampled to a fixed size of $80 \times 96$ pixels. Figure 1 shows an example image of each one of the 5 subsets in the Extended YaleB. 


\subsection{Effect of Illumination Normalisation on Identification Accuracy}

In, ${ }^{21}$ a single image per subject was selected for the gallery (i.e. the image P00A+000E+00, captured with the lightsource on the optical axis of the camera). See example gallery images of four subjects in Fig. 2b. The remaining 2376 images of the 38 persons are used as probes to test the accuracy of the recognition system. A subset of the identification results corresponding to the Haar wavelet and CityBlock distance function are shown in Tab. 2.

\begin{tabular}{|c|c|c|}
\hline Illumination Set & Angel $\theta$ & No. of Images \\
\hline Subset-1 & $\theta<12^{\square}$ & 263 images \\
Subset-2 & $20^{\square}<\theta<25^{\square}$ & 456 images \\
Subset-3 & $35^{\square}<\theta<50^{\square}$ & 455 images \\
Subset-4 & $60^{\square}<\theta<77^{\square}$ & 526 images \\
Subset-5 & $85^{\square}<\theta<128^{\square}$ & 714 images \\
\hline
\end{tabular}

Table 1: Illumination subsets in Extended Yale Face Database B.
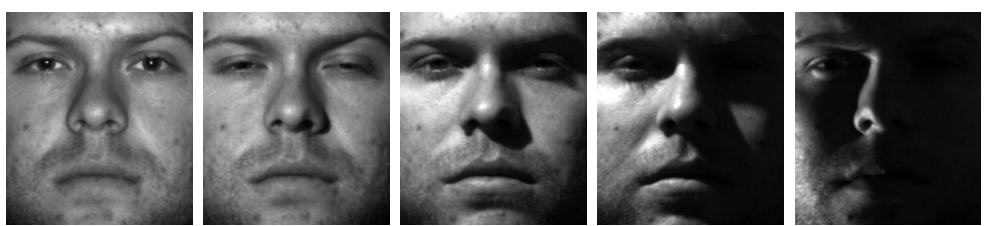

(a) Subset-1 (b) Subset-2 (c) Subset-3 (d) Subset-4 (e) Subset-5 Figure 1:

Example images of Extended Yale Face Database B.

The results show that for both LL and LH representations, applying HE on all images of the dataset reduced the overall identification error. However, a closer examination of error rates of individual illumination subsets show that HE resulted in either no reduction of identification errors or sometimes an increase in identification error of images belonging to that subset (e.g. see results for subsets-1,2\&3). We note that HE reduced the identification error of subset-3 probes when compared to using non-normalised images. However, the best result for subset-3 was achieved by the simple ZN procedure (of the feature vector), without using HE on the original cropped face images. This analysis highlight the need for an adaptive approach to HE in order to avoid undesired effects of HE when applied on reasonably well-lit images that contains little or no shadowing artifacts. To achieve this, we need to identify quantitative measures that can be used intelligently, to dynamically adapt a face recognition system to different operating conditions. Motivated by these findings in, ${ }^{21}$ we propose an image quality-based, adaptive approach to illumination normalisation by HE, as an alternative to the conventional use of HE in face recognition, where every image is first normalised, irrespective of its illumination quality. The proposed adaptive approach is described in the following section.

\begin{tabular}{|l|c|c|c|c|c|c|c|}
\hline $\begin{array}{l}\text { Wavelet } \\
\text { subband }\end{array}$ & \multicolumn{5}{|c|}{ Error Rate (\%) Vs. Illumination Subset } \\
\hline \multicolumn{2}{|c|}{$/$ Pre-process } & Set 1 & Set 2 & Set 3 & Set 4 & Set 5 & Total \\
\hline \multirow{2}{*}{$\mathrm{LL}_{2}$} & None & 8.89 & 18.20 & 83.30 & 95.82 & 97.20 & 70.71 \\
& HE & 2.67 & 25.44 & 71.65 & 91.25 & 86.83 & 65.15 \\
\hline \multirow{2}{*}{$\mathrm{LL}_{2}$ (ZN) } & None & 2.67 & 22.59 & 69.45 & 93.16 & 96.92 & 67.63 \\
& HE & $\mathbf{3 . 1 1}$ & $\mathbf{2 5 . 8 8}$ & $\mathbf{7 0 . 9 9}$ & 90.11 & 85.57 & 64.52 \\
\hline \multirow{2}{*}{$\mathrm{LH}_{2}$} & None & 8.00 & 0.00 & 30.55 & 71.10 & 95.24 & 50.97 \\
& HE & 7.56 & 0.00 & 23.08 & 55.32 & 40.62 & 29.59 \\
\hline \multirow{2}{*}{$\mathrm{LH}_{2}(\mathrm{ZN})$} & None & 7.11 & 0.00 & 10.99 & 34.22 & 66.95 & 30.47 \\
& HE & $\mathbf{7 . 5 6}$ & $\mathbf{0 . 4 4}$ & $\mathbf{1 7 . 5 8}$ & 26.62 & 14.15 & 14.31 \\
\hline
\end{tabular}




\begin{tabular}{|l|c|c|c|c|c|c|c|}
\hline \multirow{2}{*}{$\mathrm{HL}_{2}$} & None & 10.22 & 0.44 & 38.46 & 93.35 & 97.48 & 58.38 \\
& HE & 12.44 & 0.44 & 40.66 & 80.99 & 77.17 & 50.17 \\
\hline \multirow{2}{*}{$\mathrm{HL} 2$ (ZN) } & None & 8.00 & 0.00 & 11.43 & 63.88 & 96.22 & 46.00 \\
& HE & $\mathbf{8 . 4 4}$ & 0.00 & $\mathbf{1 3 . 8 5}$ & 46.96 & 66.11 & 33.71 \\
\hline
\end{tabular}

Table 2: Identification error rates (\%): illumination subsets vs. illumination normalisation.

\section{BIOMETRIC SAMPLE QUALITY MEASURES}

Real-time computation of quantitative, objective quality measures are a useful tool for biometric-based identification applications and there is a notable increase in the use of biometric quality measures in single modality recognition as well as in multimodality systems. ${ }^{7-9}$ These measures can be used at different stages of biometric based identification: as a quality control measure (e.g. to accept or reject a presented biometric sample or reacquire a new sample based on sample quality), for quality-based processing (e.g. selection of biometric modality, algorithm, system parameters) and as a confidence measure (e.g. decision reliability estimation). We adopted an existing quality index as a simple and efficient measure to quantify the illumination quality of a given face image with respect to a known reference face.

Wang and Bovik ${ }^{28}$ presented a quality measure, universal image quality index $(Q)$, applicable to various image processing applications. The universal quality index aims to provide meaningful comparisons across different types of image distortions by modelling any image distortion as a combination of three factors: loss of correlation, luminance distortion and contrast distortion. In this paper, the illumination quality of a given face image is to be defined in terms of its luminance distortion in comparison to a known reference image and we will called this the luminance quality index (LQI). The universal quality index, the LQI measure and an analysis of LQI as a suitable candidate to measure illumination quality are presented in the remainder of this section.

\subsection{Universal Quality Index}

Let $x=\left\{x_{i} \mid i=1,2, \ldots, N\right\}$ and $y=\left\{y_{i} \mid i=1,2, \ldots, N\right\}$ be the reference and the test images, respectively. The universal quality index in ${ }^{28}$ is defined as

$$
Q=\frac{4 \sigma_{x y} \bar{x} \bar{y}}{\left(\sigma_{x}^{2}+\sigma_{y}^{2}\right)\left[(\bar{x})^{2}+(\bar{y})^{2}\right]},
$$

where

$$
\begin{gathered}
\bar{x}=\frac{1}{N} \sum_{i=1}^{N} x_{i}, \quad \bar{y}=\frac{1}{N} \sum_{i=1}^{N} y_{i}, \\
\sigma_{x}^{2}=\frac{1}{N-1} \sum_{i=1}^{N} x_{i}-\bar{x}^{2}, \quad \sigma_{y}^{2}=\frac{1}{N-1} \sum_{i=1}^{N} y_{i}-\bar{y}^{2}, \\
\sigma_{x y}=\frac{1}{N-1} \sum_{i=1}^{N}\left(x_{i}-\bar{x}\right)\left(y_{i}-\bar{y}\right)
\end{gathered}
$$

Statistical features in Eq. 1 are measured locally to accommodate space variant nature of image quality and then combine them together to a single quality measure for the entire image. A local quality index $Q_{j}$ is calculated by sliding a window of size $B \times B$, pixel-by-pixel, from top-left corner until the window reaches the bottom-right corner of the image. For a total of $M$ steps, the overall quality index is given by

$$
Q=\frac{1}{M} \sum_{j=1}^{M} Q_{j}
$$

\subsection{Luminance Quality Index}

The universal quality index $Q$ can be written as a product of three components: 


$$
Q=\frac{\sigma_{x y}}{\sigma_{x} \sigma_{y}} \cdot \frac{2 \bar{x} \bar{y}}{(\bar{x})^{2}+(\bar{y})^{2}} \cdot \frac{2 \sigma_{x} \sigma_{y}}{\sigma_{x}^{2}+\sigma_{x}^{2}}
$$

The luminance quality index (LQI), which is the luminance distortion factor in $Q$ is defined as

$$
L Q I=\frac{2 \bar{x} \bar{y}}{(\bar{x})^{2}+(\bar{y})^{2}}
$$

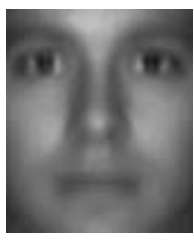

(a) Reference
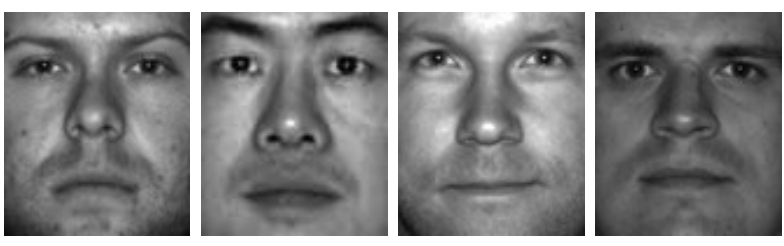

(b) Example gallery images of 4 subjects

Figure 2: The reference face image used to calculate LQI and a sample of the gallery images.

With a value range $[0,1]$, the LQI measures how close the mean luminance is between $\mathrm{x}$ and $\mathrm{y}$. LQI equals 1 , if and only if ${ }^{-} x=-y$. Global LQI (GLQI) is calculated similarly to the calculation of a single $Q$ value in Eq. 2.

Throughout this paper, the window size used is the default $8 \times 8$ window size as proposed in. ${ }^{28}$

\subsection{Evaluation of LQI as an Illumination Quality Measure}

In order to demonstrate the appropriateness of GLQI for our purposes, we determined the distribution of GLQI values in different subsets of the Extended YaleB. Since the illumination condition of each image and subset in the database is well defined, this analysis can also be used to evaluate the reliability of GLQI as an illumination quality measure. The cropped and rescaled face images from each illumination subset of the database is used for this experiment, and the reference image is the average face image (see Fig. 2a) of the 38 gallery face images (i.e. the $P 00 A+000 E+00$ image of each subject). The P00A+000E+00 image of each subject was excluded from the illumination subset- 1 since these images are used to calculate the reference image. The distribution of GLQI scores of the images in each illumination subset is shown in Fig. 3a.

The distributions of quality scores reveal that $71 \%$ of the 225 images in subset- 1 have a GLQI score of 0.95 or higher, while $92 \%$ and $80 \%$ of the images in subsets- $1 \& 2$ have a GLQI score of 0.9 or higher. Compared to subset1 , only about $30 \%$ of the images in subset- 2 have a GLQI score of 0.95 or higher. This demonstrates that the GLQI measure correctly quantified the illumination quality of images in subsets-1\&2 to be very near to that of the reference image, while also recognising that images in subset-1 are nearer to the reference image. Only $7 \%$ of images in subset-3 have a GLQI score of 0.9 or higher, while $40 \%$ of the images have a GLQI value range of [0.8, 0.9], which indicates the noticeable variation in illumination in subset-3 images. The highest GLQI score of the images selected from subset- 4 is 0.78 and nearly $74 \%$ of the images of subset- 4 has a GLQI value less than 0.6 . Nearly $43 \%$ of the images in subset-5 scored a quality value under 0.3 , while the highest GLQI score is only 0.59 . This reflects the poor illumination quality of the images in subsets-4\&5 due to the extreme changes in illumination direction (horizontally and/or vertically) with regard to the camera axis (see Tab. 1 for details) and is further evidence to the use of GLQI as a reliable illumination quality estimator.

\subsection{Effect of Illumination Normalisation on Image Quality}

We looked at how illumination normalisation affects image quality. Cropped and resampled face images from each illumination subset is used for this purpose. The P00A+000E+00 image of each subject was excluded from the illumination subset- 1 since these images were used to calculate the reference image. The illumination quality of the selected images in each subset, before and after HE was measured using GLQI. The distribution of GLQI scores of original and normalised images in each illumination subset is shown in Fig. 3. 
Compared with $92 \%$ and $84 \%$ of original images in subset- $1 \& 2$ respectively, having a GLQI score of 0.9 or higher, only $91 \%$ and $46 \%$ of images in the same 2 subsets achieved a quality score of 0.9 or higher after normalisation by HE. However, all images of subset- 3 achieved a quality score over 0.8 after $\mathrm{HE}$, while only $45 \%$ of original images in the same set had a quality score over 0.8 . However, GLQI scores of around $4 \%$ of the original images in subset-3 are higher than the highest GLQI score of images after HE. Nearly all the images of subset-4\&5 scored GLQI values over 0.8 , demonstrating that HE is a very useful illumination normalisation tool when there is a significant variation in lighting between the gallery and probe images. It is also worth

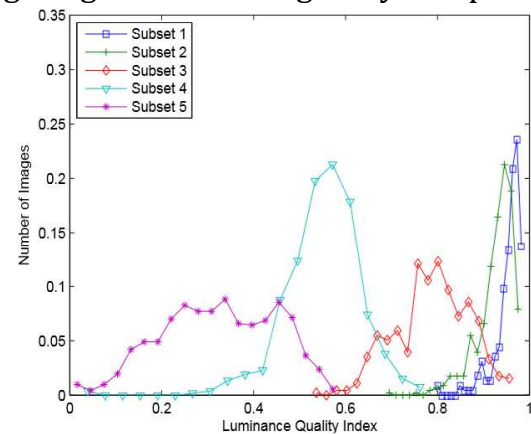

(a) GLQI scores of original images

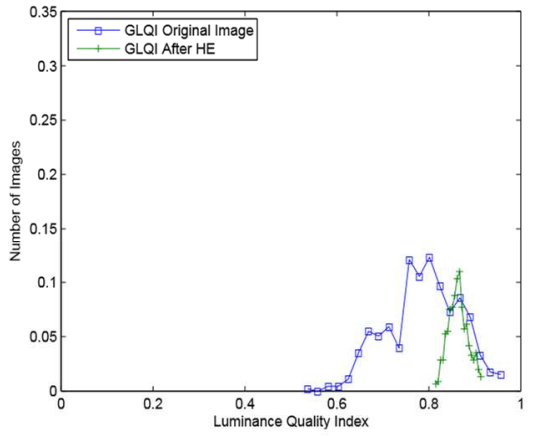

(d) Subset-3

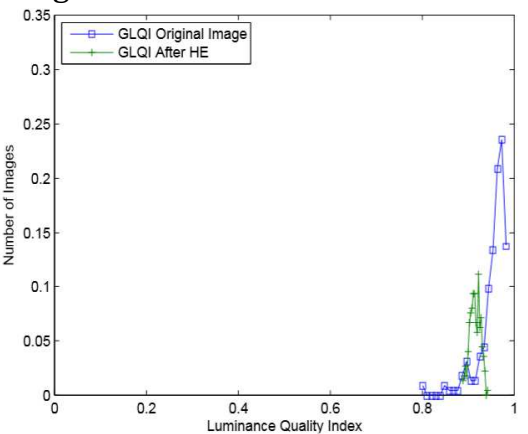

(b) Subset-1

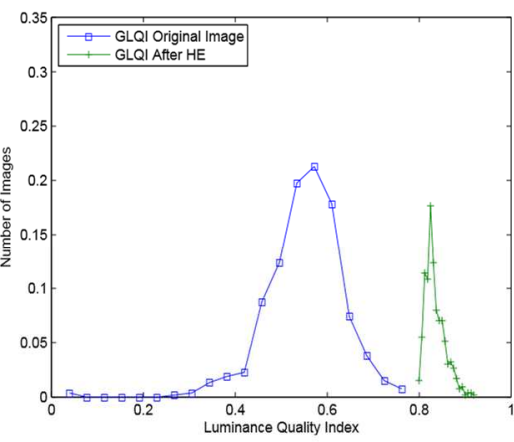

(e) Subset-4

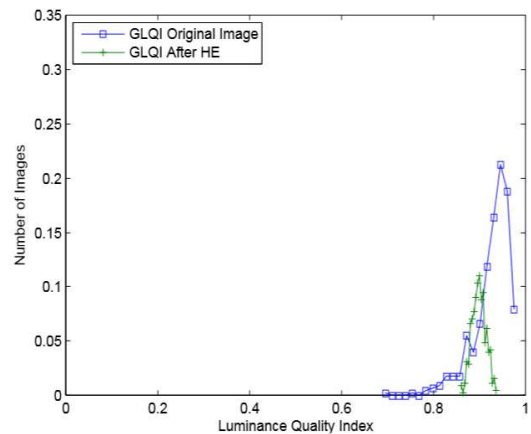

(c) Subset-2

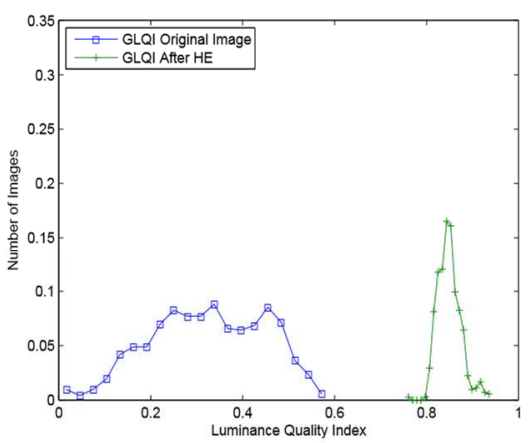

(f) Subset-5

Figure 3: Distribution of GLQI scores of the five illumination subsets (GLQI of original images and after HE).

mentioning here that the highest GLQI score of normalised images of subset-4\&5 is 0.92 and 0.94 respectively, which suggests that there is room for further improvement in illumination quality.

This assessment of image quality, before and after illumination normalisation, shows that normalising images captured under controlled lighting conditions could deteriorate image quality. The decrease in image quality due to HE correlates with the decrease in recognition accuracy when $\mathrm{HE}$ is applied to well-lit images. ${ }^{21} \mathrm{At}$ the same time, the increase in image quality seen in subsets-3,4\&5 correlates with the increase in recognition accuracy after these images are normalised. Therefore, GLQI: a quantitative measure of image quality, can be used to design an adaptive approach to illumination normalisation for face recognition.

\section{ADAPTIVE ILLUMINATION NORMALISATION}

The proposed approach uses an illumination quality measure for quality-based pre-processing. The analysis in the previous section and the efficient computation of GLQI justifies the introduction of GLQI-based adaptive normalisation. In this study, HE is selected as the pre-processing method for the adaptive normalisation, which we refer to as global quality-based adaptive HE or GQbHE. The GQbHE works by first calculating GLQI of the input image and only if its GLQI score is less than a predefined threshold (LQIT), then apply HE to the image. The threshold 
can be determined empirically depending on the objectives of the applications under consideration. For the Extended YaleB database, a sensible threshold that leads to improved GLQI value is around 0.8 GLQI.

\subsection{Identification Experiments and Results}

The affect of GQbHE and HE on identification accuracy is evaluated using the well-known Extended Yale Face Database B. The experiment setting adopted here is the same as in, ${ }^{21}$ and described in Sec. 2.3. For GQbHE, LQI score threshold values $0.7,0.75,0.8,0.85$ and 0.9 are selected based on the analysis of the distributions of LQI scores for each illumination subset (see Sec. 3.2). Identification error of different wavelet-based feature

\begin{tabular}{|c|c|c|c|c|c|c|c|}
\hline \multirow{2}{*}{\multicolumn{2}{|c|}{$\begin{array}{r}\text { Wavelet subband } \\
\text { /Pre-process }\end{array}$}} & \multicolumn{6}{|c|}{ Error Rate (\%) Vs. Illumination Subset } \\
\hline & & Set 1 & Set 2 & Set 3 & Set 4 & Set 5 & Total \\
\hline \multirow{3}{*}{$\mathrm{LL}_{2}(\mathrm{ZN})$} & None & 2.67 & 22.59 & 69.45 & 93.16 & 96.92 & 67.63 \\
\hline & HE & 3.11 & 25.88 & 70.99 & 90.11 & 85.57 & 64.52 \\
\hline & GQbHE & 2.67 & 22.81 & 69.01 & 90.11 & 84.03 & 63.05 \\
\hline \multirow{3}{*}{$\mathrm{LH}_{2}(\mathrm{ZN})$} & & 7.11 & 0.00 & 10.99 & 34.22 & 66 & 30.47 \\
\hline & HE & 7.56 & 0.44 & 17.58 & 26.62 & 14.15 & 14.31 \\
\hline & GQbHE & 7.11 & 0.00 & 11.65 & 20.34 & 11.76 & 10.94 \\
\hline \multirow{3}{*}{$\mathrm{HL}_{2}(\mathrm{ZN})$} & None & 8.00 & 0.00 & 11.43 & 63 & 96.22 & 46.00 \\
\hline & HE & 8.44 & 0.00 & 13.85 & 46.96 & 66.11 & 33.71 \\
\hline & GQbHE & 8.00 & 0.00 & 10.11 & 41.25 & 61.90 & 30.43 \\
\hline
\end{tabular}

Table 3: Recognition Error Rates: GQbHE (LQIT = 0.8) Vs. HE

representations, with HE and GQbHE (with $L Q I T=0.8$ ) are shown in Tab. 3. We also included identification error rates without a pre-processing step to normalise illumination.

Compared to the traditional use of HE, the proposed GQbHE further decreased the overall identification error by a further 1-3\%, across different feature representations. More significant reduction in errors of up to $6 \%$ can be seen for individual illumination subsets. The improvement in identification accuracy of subsets- $4 \& 5$ is quite significant. This improvement is due to the fact that the gallery images are not normalised under the GQbHE, where as traditionally, all images are normalised. These results justify the use of an adaptive approach to illumination normalisation and the use of LQI as a reliable illumination quality measure.

\section{REGION QUALITY-BASED ADAPTIVE ILLUMINATION NORMALISATION}

Shan et al. ${ }^{23}$ illustrated that a region-based approach to illumination normalisation achieves better identification accuracy rates by testing various global and region-based normalisation techniques on the Extended Yale B database. In the region-based approach, an image is first partitioned into four regions and the selected normalisation procedure (e.g. HE) is applied to each region separately. Inspired by their results and the fact that the images in the Extended YaleB exhibit regional variation in image quality as a result of the direction of the light source, we extended the adaptive illumination normalisation, presented in Sec. 4, by introducing a region qualitybased adaptive normalisation scheme. The LQI of each of the four regions (RLQI) is calculated by partitioning the local quality index map (resulting from the block-wise calculation of Eq 4) into four regions.

The distribution of GLQI and RLQI scores are shown in Fig. 4. The of RLQI scores of images in subset-3,4\&5, shown in Fig. 4c- 4e, demonstrates that although an image could be categorised as having poor illumination quality with respect to a known reference image, certain regions of that image could still be of good quality and visa-versa. The differences in the distribution of GLQI and RLQI scores of subsets-3,4\&5 highlights the appropriateness of the RLQI as a measure of illumination quality, compared to the holistic, GLQI measure. 
The proposed Region Quality-based HE (RQbHE) works by first calculating RLQI of the input image and only if its RLQI score is less than a predefined threshold (LQIT), then apply HE is applied to that region. Example images, before and after conventional and the quality-based adaptive approaches to HE are shown in Fig 5.

\subsection{Identification Experiments and Results}

The same recognition experiment is repeated to compare the two proposed adaptive approaches to $\mathrm{HE}$ with the conventional HE. For RQbHE, LQI threshold values $0.7,0.75,0.8,0.85$ and 0.9 are selected. Identification error after illumination normalisation based on HE, GQbHE (LQIT $=0.80$ ), $\mathrm{RHE}^{23}$ and RQbHE (LQIT $=0.80$ ) are shown for each subset in Tab 4. As the results indicate, the lowest overall recognition error rate, as well as the lowest error rate of majority of the illumination subsets are achieved by using the proposed adaptive normalisation, RQbHE, with $\mathrm{LH}_{2}$ as the feature representation.

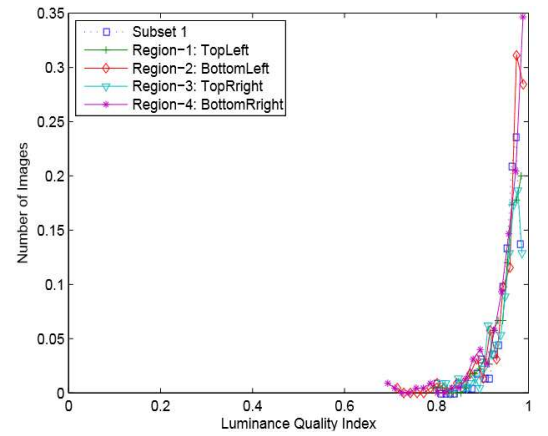

(a) Subset-1

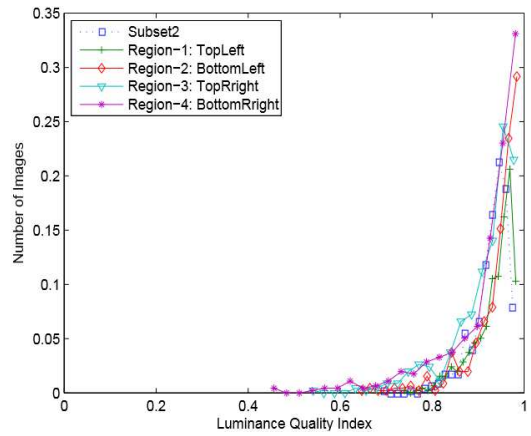

(b) Subset-2

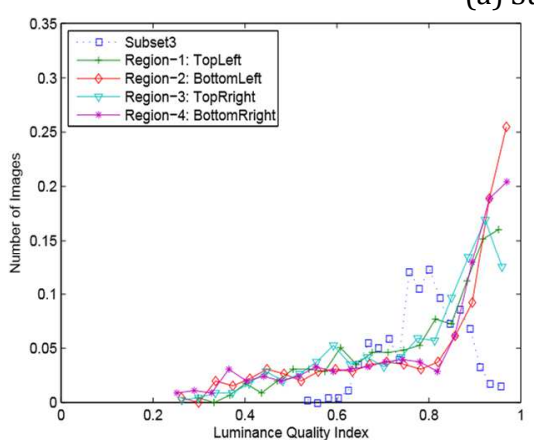

(c) Subset-3

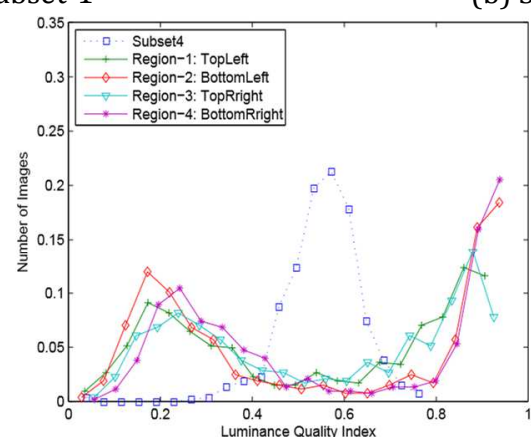

(d) Subset-4

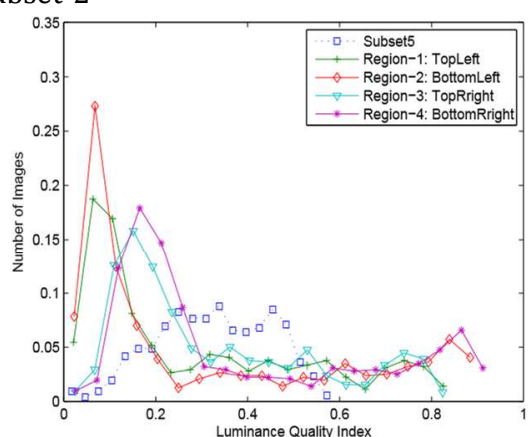

(e) Subset-5

Figure 4: Distribution of GLQI and RLQI scores of the five illumination subsets.

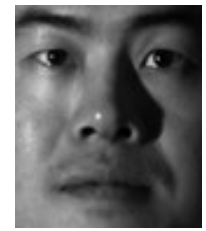

(a) Original

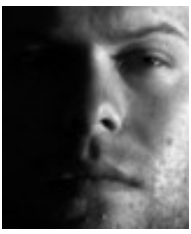

(f) Original

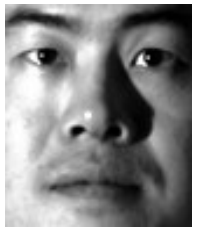

(b) HE

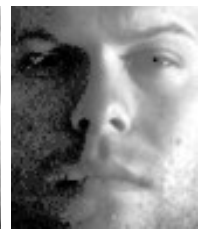

(g) HE

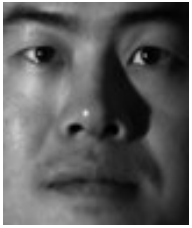

(c) GQbHE

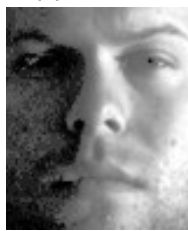

(h) GQbHE

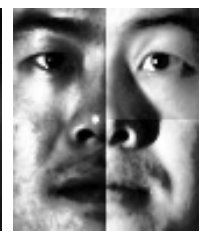

(d) RHE

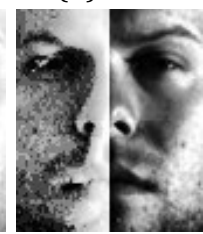

(i) RHE

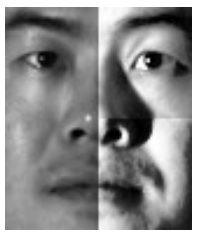

(e) RQbHE

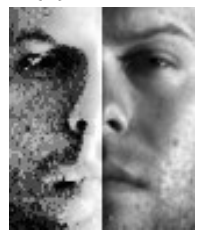

(j) RQbHE 
Figure 5: Example face images after normalisation using on HE, RHE and the proposed GQbHE and RQbHE methods

Compared to the results in Tab. 4 which uses HE for illumination normalisation, other region-based illumination normalisation techniques ${ }^{23}$ and recently published face recognition algorithms such as local binary patterns (LBP) and local ternary patterns (LTP) ${ }^{29}$ have achieved better identification accuracy rates on the Extended Yale B. This could be due to the robustness of the face descriptor as well as the pre-processing chain used to normalise illumination. We are currently investigating the use of LQI-based adaptive illumination normalisation

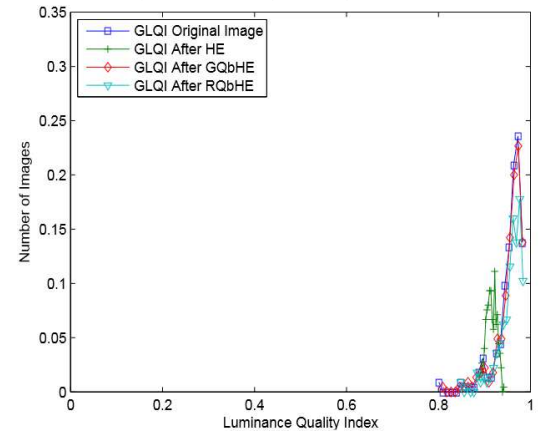

(a) Subset-1

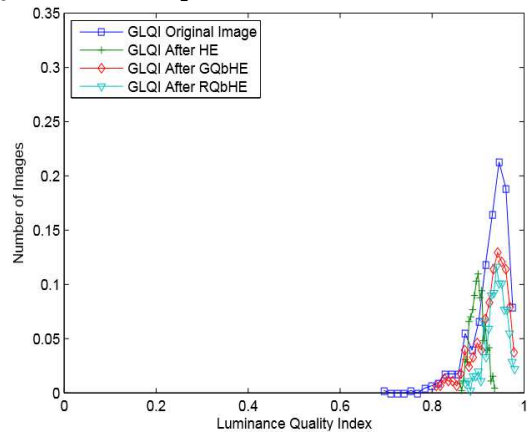

(b) Subset-2

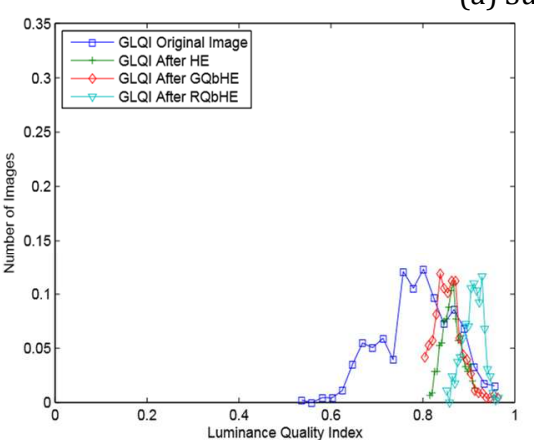

(c) Subset-3

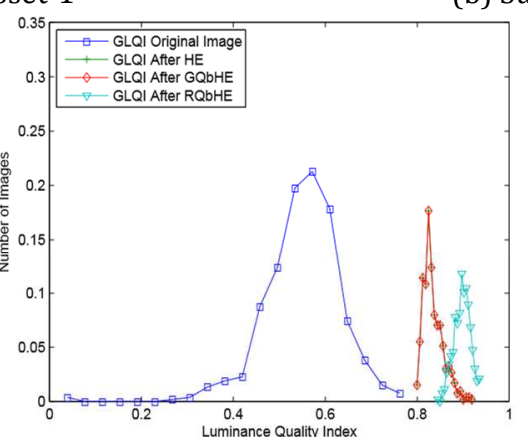

(d) Subset-4

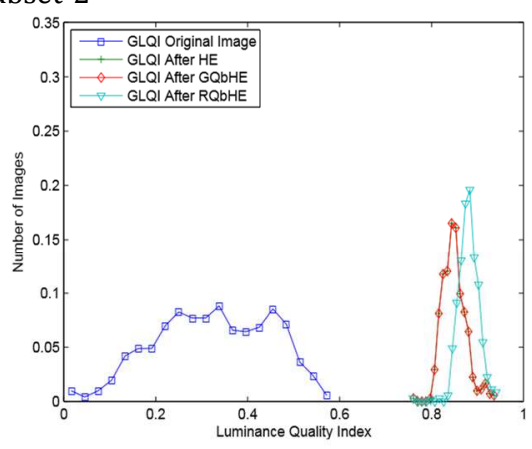

(e) Subset-5

Figure 6: Distribution of GLQI scores of the five illumination subsets after HE, GQbHE and RQbHE.

for different pre-processing techniques as well as the effects of the proposed adaptive normalisation on different face feature representations.

\section{CONCLUSIONS AND FUTURE WORK}

A common approach to address the effect of varying lighting conditions on the accuracy of face recognition systems is to pre-process the face image sample in order to normalise the illumination. Histogram equalisation is a widely used normalisation technique in face recognition. However, it has been shown that applying HE on well-lit face images could lead to a decrease in recognition accuracy. This paper presents a quality-based adaptive approach to illumination normalisation for face recognition. Illumination quality of a face image is measured in terms of its luminance distortion, using an efficient quality index that measures how close the mean luminance is between a probe image and a predefined reference image. In the proposed global quality-based HE or GQbHE, HE is applied on a probe image only if its luminance distortion is higher than a predefined threshold. The GQbHE is extended to a region quality-based HE, or RQbHE, where each $4 \times 4$ region of an image is normalised only if the region-LQI score is less than a predefined threshold.

We tested the effect of the proposed adaptive approaches and the conventional use of HE, on identification accuracy using the Extended Yale Face Database B, which consists of images captured under controlled as well as 
extreme lighting conditions. Experimental results demonstrate that the proposed illumination quality based, adaptive approaches lead to significant improvements in recognition accuracy when compared to the use of corresponding non-adaptive illumination normalisation scheme.

The LQI measure could also be used to select fusion parameters for the multi-stream face recognition. ${ }^{19-21}$ For example, a higher weight could be given to the approximation subband when probe images are captured under well-controlled lighting conditions, while a higher weight is given to the detail subbands (e.g. LH, HL)

\begin{tabular}{|c|c|c|c|c|c|c|c|}
\hline \multicolumn{8}{|c|}{ xtended Yale Database B: Identification Error Rates } \\
\hline \multirow{2}{*}{\multicolumn{2}{|c|}{$\begin{array}{r}\text { Wavelet subband } \\
\text { /Pre-process }\end{array}$}} & \multicolumn{6}{|c|}{ Error Rate (\%) Vs. Illumination Subset } \\
\hline & & \multirow{2}{*}{$\begin{array}{r}\text { Set 1 } \\
2.67\end{array}$} & \multirow{2}{*}{$\begin{array}{l}\text { Set } 2 \\
22.59\end{array}$} & \multirow{2}{*}{$\frac{\text { Set } 3}{69.45}$} & \multirow{2}{*}{$\begin{array}{l}\text { Set } 4 \\
93.16\end{array}$} & \multirow{2}{*}{$\begin{array}{l}\text { Set } 5 \\
96.92\end{array}$} & Total \\
\hline \multirow{5}{*}{$\mathrm{LL}_{2}(\mathrm{ZN})$} & None & & & & & & 67.63 \\
\hline & & 3.11 & 25.88 & 70.99 & 90.11 & 85.57 & 64.52 \\
\hline & GQbHE & 2.67 & 22.81 & 69.01 & 90.11 & 84.03 & 63.05 \\
\hline & $\mathrm{HE}$ & 0.00 & 0.00 & 30.77 & 79.47 & 81.79 & 48.06 \\
\hline & RQbHE & 3.56 & 19.30 & 42.86 & 76.62 & 78.85 & 52.90 \\
\hline \multirow{5}{*}{$\mathrm{LH}_{2}(\mathrm{ZN})$} & 1 & .11 & .00 & 10 & 34.22 & 66.95 & 0.47 \\
\hline & & & 0.44 & & & & \\
\hline & GQbHE & 7.11 & 0.00 & 11.65 & 20.34 & 11.76 & 10.94 \\
\hline & HE & 7.56 & 0.00 & 16.70 & 24.14 & 13.45 & 13.30 \\
\hline & RQbHE & 7.11 & 0.00 & 13.63 & 18.25 & 9.94 & 10.31 \\
\hline \multirow{5}{*}{$\mathrm{HL}_{2}(\mathrm{ZN})$} & IVore & 8.0 & 0.00 & 11.43 & 63.88 & 96.22 & 46 \\
\hline & $\mathrm{HE}$ & 8.44 & 0.00 & 13.85 & 46.96 & 66.11 & 33.71 \\
\hline & GQbHE & 8.00 & 0.00 & 10.11 & 41.25 & 61.90 & 30.43 \\
\hline & RHE & 9.78 & 0.00 & 13.85 & 47.53 & 65.69 & 33.84 \\
\hline & RQbHE & 8.00 & 0.00 & 10.11 & 41.25 & 61.90 & 30.43 \\
\hline
\end{tabular}

Table 4: Recognition error rates for the Extended Yale B Database: RQbHE Vs. GQbHE

when probe images are captured under poor lighting conditions. Future work involves the integration of LQI into a holistic quality measure that takes into account the changes in facial expressions and head pose during enrolment and test stages.

\section{ACKNOWLEDGMENTS}

The authors would like to acknowledge Mr. Ali J. Abboud's current study on objective image quality measures as a part his of PhD.

\section{REFERENCES}

1. M. Turk and A. Pentland, "Eigenfaces for Recognition," Journal of Cognitive Neuroscience 3(1), pp. 71-86, 1991.

2. P. N. Belhumeur, J. P. Hespanha, and D. J. Kriegman, "Eigenfaces vs. Fisherfaces: Recognition UsingClass Specific Linear Projection," IEEE Transactions on Pattern Analysis and Machine Intelligence 19, pp. 711-720, July 1997.

3. K. Etemad and R. Chellappa, "Discriminant analysis for recognition of human face images," Journal of the Optical Society of America A 14, pp. 1724-1733, August 1997.

4. M. S. Bartlett, L. H. Martin, and T. J. Sejnowski, "Independent component representation for face recognition," in Conference on Human Vision and Electronic Imaging III, Proc. of the SPIE, pp. 528-539, 1998.

5. R. Brunelli and T. Poggio, "Face Recognition: Feature vs. Templates," IEEE Transactions on Pattern Analysis and Machine Intelligence 15, pp. 1042-1053, October 1993. 
6. L. Wiskott, J.-M. Fellous, N. Kr"uger, and C. von der Malsburg, "Face Recognition by Elastic Bunch Graph Matching," in Computer Analysis of Images and Patterns, CAIP'97, Kiel, G. Sommer, K. Daniilidis, and J. Pauli, eds., Proc. 7th Int'l Conf(1296), pp. 456-463, 1997.

7. K. Kryszczuk, J. Richiardi, P. Prodanov, and A. Drygajlo, "Error Handling in Multimodal Biometric Systems using Reliability Measures," in 12th European Conference on Signal Processing, in Proc. EUSIPCO, September 2005.

8. C. Belcher and Y. Du, "Information Distance Based Contrast Invariant Iris Quality Measure," in Mobile Multimedia/Image Processing for Military Security and Applications, Proc. SPIE 6982, p. 698200, April 2008.

9. P. Grother and E. Tabassi, "Performance of biometric quality measures," IEEE Transactions on Pattern Analysis and Machine Intelligence 29(4), pp. 531-543, 2007.

10. A. S. Georghiades, D. J. Kriegman, and P. N. Belhumeur, "Illumination cones for recognition under variablelighting: faces," in Proc. IEEE Computer Vision and Pattern Recognition, pp. 52-58, 1998.

11. A. S. Georghiades, P. N. Belhumeur, and D. J. Kriegman, "From few to many: Illumination cone modelsfor face recognition under variable lighting and pose," IEEE Transactions on Pattern Analysis and Machine Intelligence 23(6), pp. 643-660, 2001.

12. T. Okabe and Y. Sato, "Object recognition based on photometric alignment using RANSAC," in Computer Vision and Pattern Recognition, Proc. IEEE Conf. 1, pp. 221-228, June 2003.

13. K.-C. Lee, J. Ho, and D. J. Kriegman, "Acquiring Linear Subspaces for Face Recognition under Variable Lighting," IEEE Transactions on Pattern Analysis and Machine Intelligence 27, pp. 684-698, May 2005.

14. P. W. Hallinan, "A low-dimensional representation of human faces for arbitrarylighting conditions," inComputer Vision and Pattern Recognition, Proc. IEEE Conf., pp. 995-999, June 1994.

15. A. Shashua, “On Photometric Issues in 3D Visual Recognition from a Single 2D Image," International Journal of Computer Vision 21, pp. 99-122, January 1997.

16. P. N. Belhumeur and D. J. Kriegman, "What is the set of images of an object under all possible lightingconditions?," in Computer Vision and Pattern Recognition, Proc. IEEE Conf. 00, pp. 270-277, 1996.

17. J. H. Lai, P. C. Yuen, and G. C. Feng, "Face recognition using holistic Fourier invariant features," Pattern Recognition 34, pp. 95-109, 2001.

18. J.-T. Chien and C.-C. Wu, "Discriminant Waveletfaces and Nearest Feature Classifiers for Face Recognition," IEEE Transactions on Pattern Analysis and Machine Intelligence 24, pp. 1644-1649, December 2002.

19. H. Sellahewa, Wavelet-based Automatic Face Recognition for Constrained Devices. PhD thesis, University of Buckingham, Buckingham, UK, 2006.

20. S. A. Jassim and H. Sellahewa, "Multi-stream Face Recognition on dedicated mobile devices for CrimeFighting," in Mobile Multimedia/Image Processing for Military and Security Applications, Proc. SPIE 6402, pp. 6402-24, September 2006.

21. H. Sellahewa and S. A. Jassim, "Illumination and Expression Invariant Face Recognition: Toward SampleQualitybased Adaptive Fusion," in Biometrics: Theory, Applications and Systems, Proc. Second IEEE Int'l. Conf., Septempber 2008.

22. H. Wang, S. Z. Li, and Y. Wang, "Face recognition under varying lighting conditions using self quotientimage," in Automatic Face and Gesture Recognition (AFGR), Proc. 6th IEEE Int. Conf, p. 819, 2004.

23. S. Shan, W. Gao, B. Cao, and D. Zhao, "Illumination Normalization for Robust Face Recognition AgainstVarying Lighting Conditions," IEEE International Workshop on Analysis and Modeling of Faces and Gestures , pp. 157164, 2003.

24. R. Gross and V. Brajovi, "An Image Preprocessing Algorithm for Illumination Invariant Face Recognition,"in Audio-and Video-Based Biometric Person Authentication, Proc. 4th Int'l Conf. AVBPA, pp. 11-18, June 2003.

25. S. G. Mallat, "A Theory for Multiresolution Signal Decomposition: The Wavelet Representation," IEEE Transactions on Pattern Analysis and Machine Intelligence 11, pp. 674-693, July 1989.

26. I. Daubechies, Ten lectures on wavelets, Society for Industrial and Applied Mathematics, Philadelphia, PA, USA, 1992.

27. H. Sellahewa and S. Jassim, "Wavelet-based face verification for constrained platforms," in Biometric Technology for Human Identification II, Proc. SPIE 5779, pp. 173-183, March 2005. 
28. Z. Wang and A. C. Bovik, "A Uiversal Image Quality Index," IEEE Signal Processing Letters 9, pp. 81-84, march 2002.

29. X. Tan and B. Triggs, "Enhanced Local Texture Feature Sets for Face Recognition Under Difficult LightingConditions," in Analysis and Modelling of Faces and Gestures, LNCS 4778, pp. 168-182, 2007. 